\title{
Skin attachable flexible sensor array for respiratory monitoring
}

Eugen Koch, Andreas Dietzel, TU Braunschweig Institut für Mikrotechnik, Alte Salzdahlumer Str. 203, 38124 Braunschweig, Deutschland, eugen.koch@tu-bs.de

\begin{abstract}
Flexible electronic systems became increasingly popular in recent years. They usually can be bent or even stretched while fully maintaining their functionality opening up a wide field of various new applications. In this paper a novel $6 \times 6$ sensor array for curvature sensing in the format of a thin flexible polyimide foil is introduced. The sensor foil is to be used for respiratory monitoring of premature infants by directly attaching it to the skin for measuring the body deformations caused by breathing. Sensor signals shall in future be used not only to trigger the respirator but also to provide time dependent body surface reconstruction as a diagnostic tool. One single sensor element consists of four gold strain gauges in a Wheatstone bridge configuration. For suppressing sensor response to foil stretching and for increasing bending sensitivity we introduced a double-sided sensor design with strain gauges on both surfaces of the thin foil leading to a $170 \%$ higher sensitivity than a one-sided sensor design. The complete sensor array foil of less than $20 \mu \mathrm{m}$ in thickness can be fabricated without flipping the substrate. Fully functional sensor foils were characterized with respect to sensitivity, dependence between bending orientation and sensor matrix output signals. Double sided sensor elements with different orientations are arranged in an alternating pattern across the array allowing to fully and unambiguously determine the bending vector utilizing plausibility considerations on basis of signals from neighboring elements. A small initial bending resulting from fabrication induced stresses was observed but could easily be compensated in digital sensor signal analysis. In summary, our first tests of this novel sensor array together with the scalability of implemented fabrication processes are very promising and meet the fundamental criteria to be used as sensor array for respiratory monitoring of infants.
\end{abstract}

\section{Introduction}

In recent years, electronic systems with attributes like "flexible", "stretchable", "elastic" and "wearable" became increasingly popular in the world of fascinating new technologies. Ultra-thin, lightweight, unbreakable and low cost producible electronic products which can be bent and often even be stretched, while fully maintaining their functionality, allow easy attachment to bodies with non-planar shapes and therefore enable a variety of new applications [1-5].Their popularity is supported by a continuous development of new materials and fabrication technologies [6-11] opening new opportunities in designing these systems. Polymer materials like polyimide (PI), polyethylene terephthalate (PET), polyethylene naphthalate (PEN), parylene and polydimethylsiloxane (PDMS) play a key role in this development. They usually serve as carrier substrate and insulating layer for the electronic parts with the desired flexibility or even stretchability. They can even be partially turned into conductors by mixing with conductive particles like carbon nano tubes [12]. Developments in fabrication technologies for patterning, deposition, coating, lamination and testing of electronic systems are as important as advancements in used materials. A potential mass fabrication option is the suitability for so-called roll-to-roll processing (R2R), which enables fast, large-area and therefore costeffective electronic fabrication. Applications can be found in the consumer electronics sector in form of flexible mobile phones, displays and touch screens; in the fast growing sector of RFID (radio-frequency identification)technology for food monitoring, transportation, logistic and passports; in the structural-health monitoring sector allowing inspection of difficult to access objects and bodies with non-planar shapes; in the wearable electronics sector, for fitness tracking, as communication device and navigation tool; and in the medical fields, e.g. for monitoring various vital parameters of the human body, like heartbeat, body temperature, blood pressure and respiratory rate.

The major motivation for our work comes from the targeted application as a flexible sensor patch for the monitoring and triggering of artificial respiration for premature infants as illustrated in Figure 1. 


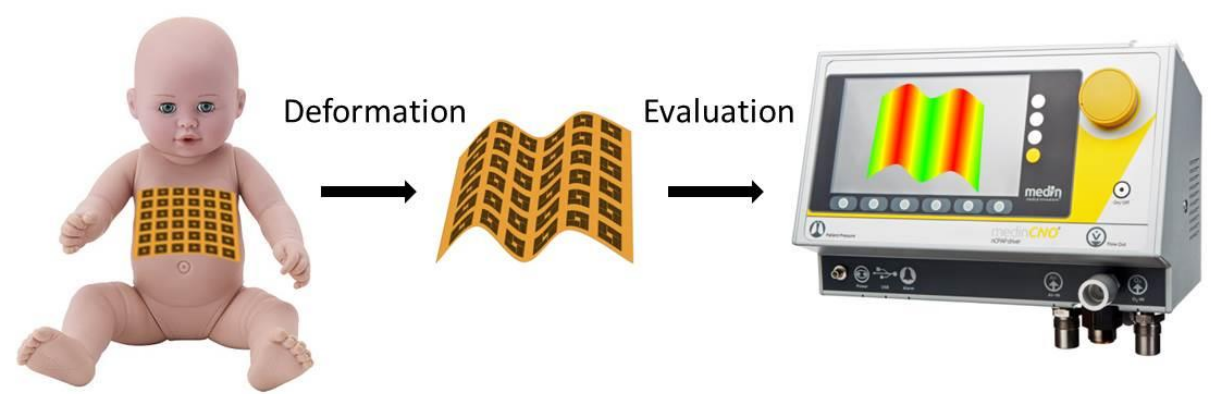

Figure 1: Illustration of the concept for monitoring and triggering artificial respiration for premature infants utilizing a skin attachable flexible sensor array

A sensor array attached to the skin at the transition between thorax and belly will by breathing of the infant experience an oscillating deformation, which can be detected by the sensor array. The sensor information can primarily be used to detect the beginning and ending of a respiration cycle and hence to trigger the respirator. A further goal is to reconstruct the surface of the covered body using the sensor array signals. This new surface information can be recorded over a specific time period and subsequently be analyzed to detect dynamic patterns in breathing, which can be used to deduce diagnostic information and to improve the medical treatment. A difference in lifting between the left and the right lung, which is a symptom of a known disease of infants [13] could eventually be detectable.

The aim is to build up a sensor array which has mechanical properties similar to the human skin and adapts perfectly to the surface topography of the body causing minimum stress to the infant. Our new skin attachable sensor array is developed in the form of a flexible foil but can in future work be transformed into a stretchable design to even better fulfill the requirements for the mentioned application as a respiratory monitoring tool. Further challenges are given by the architecture of the sensor network requiring appropriate wiring for fast readout and for current-saving operation e.g. for wireless applications. Thin ribbons of single-crystalline silicon on plastic substrates were already used showing high deformation sensitivity [14]. However, advantages of a concept based on metal sensors could probably be found in lower temperature sensitivity, higher maximum elongations, no influence on crystal orientation when orienting sensors in different directions, lower processing temperatures and an easy scalable fabrication leading to lower production costs.

\section{Design of the sensor array}

Figure 2 (a) shows a fabricated sensor array on a glass wafer with $6 \times 6=36$ sensor elements and in total 74 conductor lines to be connected to the evaluation electronics (72 measurement lines and 2 common mode power supply lines). Each sensor element consists of four metal strain gauges in a Wheatstone bridge configuration as illustrated by the magnified view of a single sensor element in Figure $\mathbf{2}$ (b). To achieve a better sensitivity we designed so called "double-sided" sensor elements. Figure $\mathbf{2}$ (c) shows the resistor arrangement on both sides of the foil substrate and Figure $\mathbf{2}$ (d) shows the connection of the four resistors to a full Wheatstone bridge configuration. Two resistors are placed on the top side of the foil substrate and two others on the bottom side. 


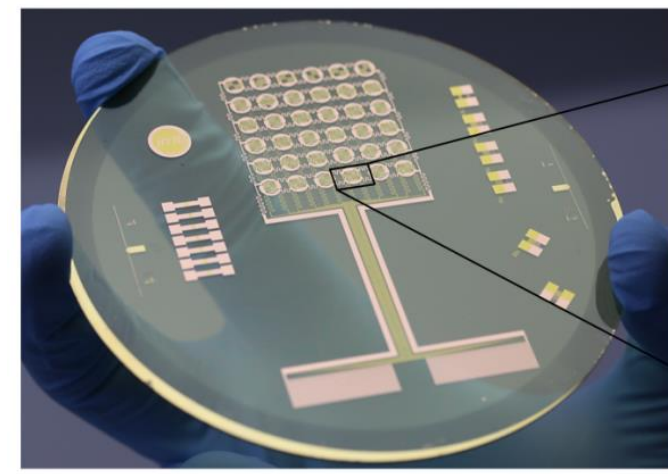

a.

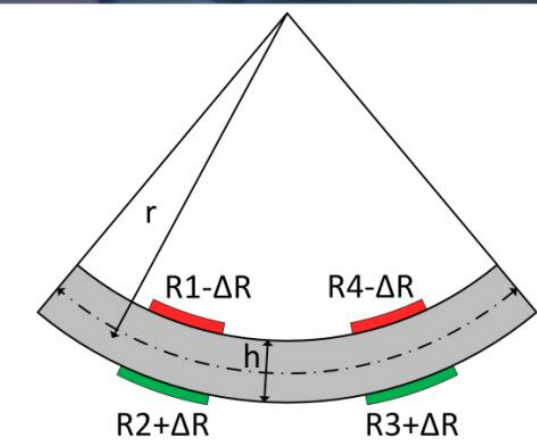

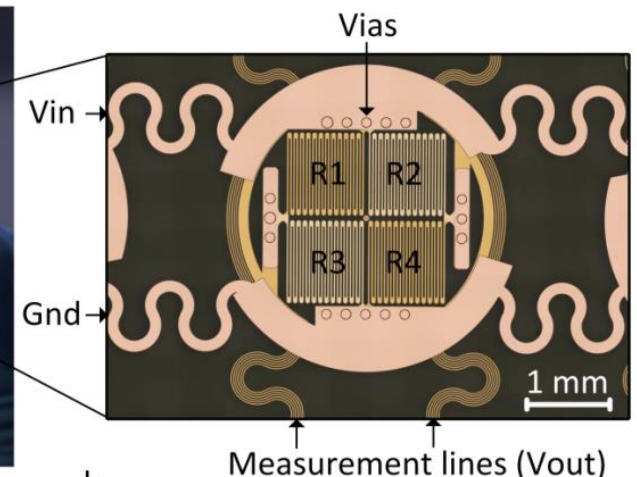

b.

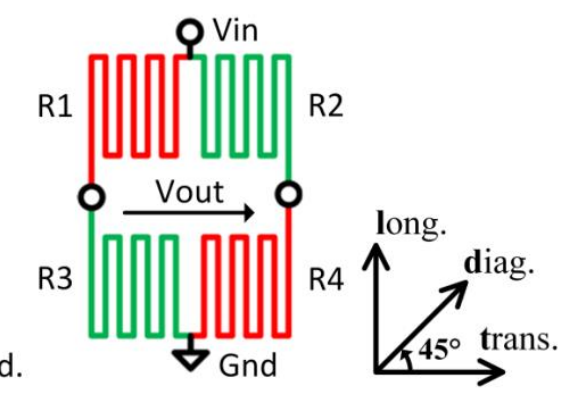

Figure 2: (a) Fabricated $6 \times 6$ sensor array still residing on a glass wafer, (b) magnification of a single sensor element in a Wheatstone bridge configuration, (c) schematic illustration of the double-sided resistor arrangement on foil substrate, (d) schematic illustration of implemented full Wheatstone bridge configuration.

The output signal of the Wheatstone bridge is given by:

$$
V_{\text {out }}=V_{\text {in }} \cdot\left(\frac{R_{4}}{R_{2}+R_{4}}-\frac{R_{3}}{R_{1}+R_{3}}\right)
$$

In an undeformed state all four resistors should have the same value $R_{i}=R,(i=1 \ldots 4)$ and the output signal $V_{\text {out }}$ equals zero. Bending of the foil leads to elongations of opposite directions $\varepsilon_{1}=\varepsilon_{4}=-\varepsilon$ and $\varepsilon_{2}=\varepsilon_{3}=\varepsilon$ which induce changes in resistance as $R_{i}=R+\Delta R_{i}$. By inserting $R_{i}$ in equation (1) and with the assumption that $R \gg \Delta R_{i}$ we get:

$$
V_{\text {out }} \approx \frac{V_{\text {in }}}{4} \cdot\left(\frac{\Delta R_{1}}{R}+\frac{\Delta R_{2}}{R}+\frac{\Delta R_{3}}{R}+\frac{\Delta R_{4}}{R}\right)
$$

The relation between elongation $\varepsilon_{i}$ and change in resistance $\frac{\Delta R_{i}}{R}$ is given by the gauge factor GF according to:

$$
\frac{\Delta R_{i}}{R}=G F \cdot \varepsilon_{i}
$$

From (2) and (3) we derive:

$$
\mathrm{V}_{\text {out }} \approx \mathrm{GF} \cdot \mathrm{V}_{\mathrm{in}} \cdot \varepsilon
$$

Assuming $G F=2$ for metal conductors, a bending radius of $r$ and a foil thickness $h$ reveals

$$
\mathrm{V}_{\text {out }} \approx \mathrm{V}_{\mathrm{in}} * \frac{\mathrm{h}}{\mathrm{r}}
$$


for the double-sided design which is twice as high as for the one-sided design. In practice however, the sensors show non negligible "transverse sensitivity" in two of the four one-sided sensor elements which leads to an additional decrease in output signal. Besides higher sensitivity a double-sided sensor design has further advantages. The sign of the output signal unambiguously tells us the polarity of curvature whereas in a onesided sensor design the sign already changes between longitudinal and transversal bending. Double sided sensor elements only react to curvatures; simple stretching would lead to the same change in resistance in all four strain gauges and therefore wouldn't affect the output signal.

For reconstructing the surface topography, the orientation and the amount of curvature at each measurement point (location of the sensor element) has to be measured and subsequently one continuous surface can be obtained by interpolation. Strictly speaking, unambiguous identification of the curvature vectors requires Wheatstone sensor bridges in at least three different orientations at each measurement point. This is for single measurement points realized in so called strain gauge rosettes which are often used in structural health monitoring to determine the three independent components in a plane strain. However, the need of three Wheatstone bridges on each measurement point in the sensor array would lead to a rather complicated fabrication process. In an alternative solution single sensor elements in each measurement point are sufficient if they are arranged in different directions in an alternating pattern across the array (according to Figure $\mathbf{3}$ ).

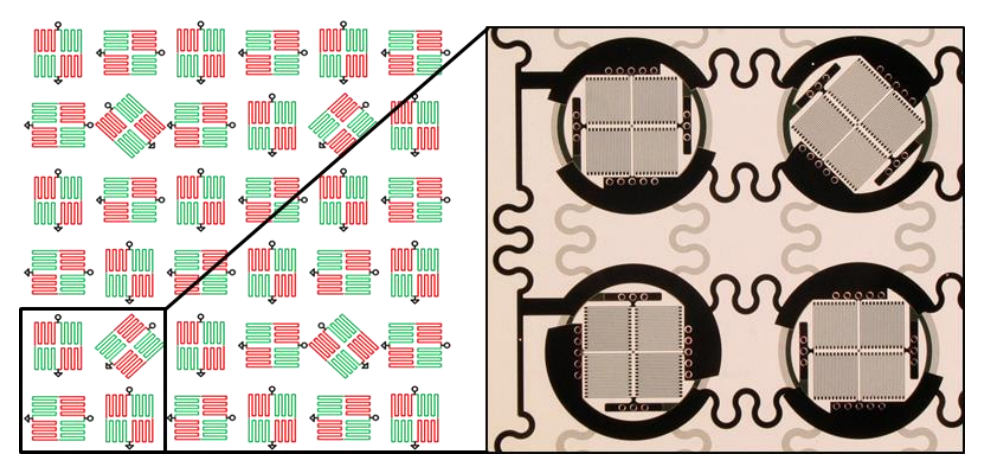

Figure 3: Sensor array with alternating sensor orientations

To still be able to identify the orientation and amount of curvature at each measurement point, plausibility considerations with help of the neighboring measurement points can be applied. Plausible assumptions can only be applied if bending only slightly changes between neighbors; in other words the sensor density has to be matched with the measurement task.

\section{Micro fabrication of flexible sensor array}

The schematic in Figure 4 shows a cross section of our double-sided sensor design with indications of the seven major process steps and used materials. 


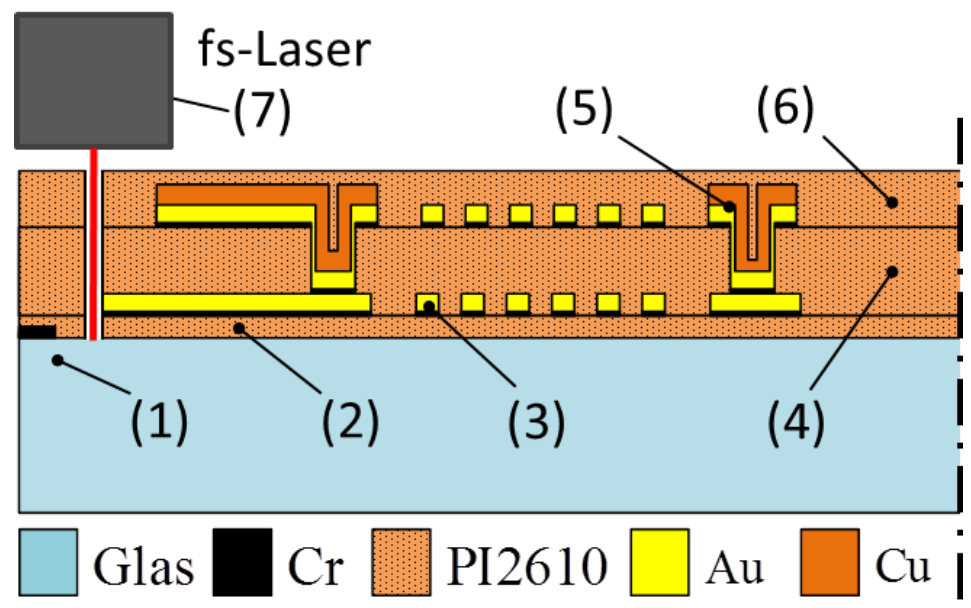

Figure 4: Schematic illustrating the double-sided sensor cross section. The major process steps associated with certain structure elements are (1) Substrate, (2) bottom layer (polyimide spin-coating), (3) first sensor layer (metal deposition and lithographic structuring), (4) interlayer (polyimide spin-coating), (5) second sensor layer (metal deposition, lithographic structuring and partial copper electroplating), (6) top layer (polyimide spin-coating) and (7) foil shape and release (defined by fs-laser ablation cutting)

(1) Substrate: As carrier substrates $\varnothing 4$ inch glass wafers are used which are first cleaned in a spray processor to avoid particle contamination and to ensure a better release of the sensor array after fabrication. Next, a thin ring of chromium at the edge of the glass wafer is applied to improve the adhesion of the subsequent polyimide layer to the carrier. Without this ring the polyimide tends to release from glass wafer before the fabrication process is finished.

(2) Bottom layer: For the bottom layer $1 \mathrm{ml}$ of the polyimide PI-2610 from HD MicroSystems is spincoated at $3000 \mathrm{rpm}$. Subsequently the polyimide is annealed by heating with a ramp from $90^{\circ} \mathrm{C}$ to 220 ${ }^{\circ} \mathrm{C}$ and kept at that temperature for $10 \mathrm{~min}$ which according to the datasheet is sufficient to achieve an almost complete polymerization. The final thickness of this layer is $\approx 2.5 \mu \mathrm{m}$.

(3) First sensor layer: For the first sensor layer a $40 \mathrm{~nm}$ thin $\mathrm{Au}$ layer with an underlying adhesion promoting $\mathrm{Cr}$ layer of $10 \mathrm{~nm}$ is sputtered. $\mathrm{Au} / \mathrm{Cr}$ is structured by standard lithography. Before wet etching an $\mathrm{O}_{2}$-plasma treatment for $5 \mathrm{~min}$ is applied to activate and to render the surface hydrophilic. This step ensures that otherwise non-wetting edges will also be etched.

(4) Interlayer: According to equation (5) the thickness of this layer has a direct influence on the sensitivity. To get a total thickness of $\approx 11.5 \mu \mathrm{m}$ we successively apply two layers of the polyimide PI2610 at a spin speed of $1200 \mathrm{rpm}$. The first polyimide layer has to receive a soft bake of $90 \mathrm{~s}$ at $90^{\circ} \mathrm{C}$ and $90 \mathrm{~s}$ at $150^{\circ} \mathrm{C}$ before the second layer can be coated. Subsequently the complete polyimide layer is annealed by heating with a ramp from $90{ }^{\circ} \mathrm{C}$ to $220^{\circ} \mathrm{C}$ and kept for $10 \mathrm{~min}$ at the final temperature. Next, vias (see Figure $\mathbf{2}(\mathbf{b})$ ) for the interconnection between first and second sensor layer are etched in a barrel etcher with following parameters: duration: $22 \mathrm{~min}, \mathrm{O}_{2}$-gasflow: $144 \mathrm{sccm}, \mathrm{CF}_{4}$-gasflow: 6 sccm, chamber pressure: 150 mTorr, etch mask: aluminum.

(5) Second sensor layer: As for the first sensor layer, a 40/10 $\mathrm{nm}$ thin $\mathrm{Au} / \mathrm{Cr}$ layer is sputtered. A further layer is applied by electroplating $1.5 \mu \mathrm{m}$ thick copper. Before electroplating areas for the sensor elements are protected with photoresist to avoid a growth of copper at these points. The copper lowers the resistance of the supply lines and vias to avoid high voltage drops which would decrease the sensitivities. Finally, an $\mathrm{O}_{2}$-plasma is applied before all three metal layers are structured by a standard wet etching process.

(6) Top layer: A $2.5 \mu \mathrm{m}$ thin polyimide layer is applied as described in step (2) but here uncovering of the contact pads for the evaluation electronic is necessary which is made by applying a simple adhesive foil above the pads before polyimide spin-coating. This foil can be easily removed directly after coating while the polyimide is still liquid. The last step is a hard bake of the whole sensor with a ramp from 90 ${ }^{\circ} \mathrm{C}$ to $250^{\circ} \mathrm{C}$ for $60 \mathrm{~min}$. 
(7) Foil shape: The final foil shape is defined by cutting using a femtosecond-laser. This has two major advantages compared with a manual cutting by a scalpel or scissor. On the one hand we can easily apply smooth radii to the corners of our design leading to more stability against fracture. On the other hand we can better define exact dimensions when cutting the contact pads allowing better control of alignment to the electronic connector. After laser cutting we easily can peel off the flexible sensor array foil from the rigid glass wafer.

For the fabrication of single sensor elements used in first tests we applied a slightly different process in which the photo definable SU-8 3000 series from MicroChem was used for the interlayer with a total thickness of 50 $\mu \mathrm{m}$. We finally switched to polyimide because the SU-8 gets brittle after some weeks and tends to break at small bending radii.

\section{Sensor functionality and discussion}

To experimentally proof the advantage of a double-sided sensor design, one-sided and double-sided single sensor elements were fabricated with SU-8 and tested in bending experiments [15]. Figure 5 shows the offset compensated output signal $\mathrm{V}_{\text {out }}$ against the bending radius in a semi logarithmic plot. According to the definition given in Figure $\mathbf{2}$ (d) we performed bending experiments in longitudinal (I), diagonal (d) and transversal $(t)$ orientation. The signs $(+/-)$ behind the orientations $(I, d, t)$ represent the bending polarity realized by simply flipping the sensor before bending. The dotted lines in both plots represent calculated values for $V_{\text {out }}$ in longitudinal direction based on the following parameters: amplification $A=0$, interlayer thickness $\mathrm{h}=50 \mu \mathrm{m}$, gauge factor $\mathrm{GF}=2$ and a supply voltage of $\mathrm{V}_{\mathrm{in}}=5 \mathrm{~V}$.
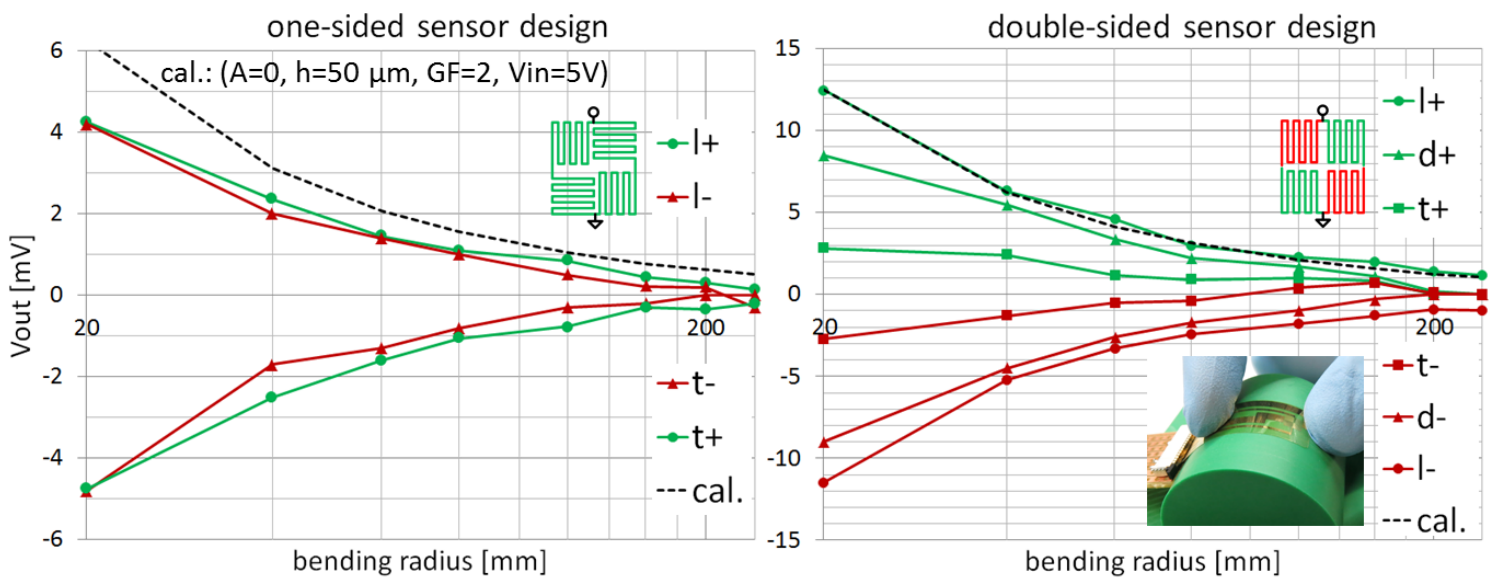

Figure 5: Bending measurement results for a one-sided sensor (left) and a double-sided sensor (right) (l=longitudinal, $t=$ transversal and $d=$ diagonal bending orientations, $+/$-=bending polarity, cal. =calculated values for $1+$. Note that the signs of $\mathrm{l}$ - and $\mathrm{t}$ - for the one-sided sensor were turned for easier comparison. Inserted picture: measurement setup.

Calculated and measured values agree very well for the double-sided sensor design whereas for the one-sided sensor design a difference is observed. This can be explained by a "transverse sensitivity" of our strain gauges differing from zero. Commercial strain gauges usually have a transverse sensitivity of less than $1 \%$ but in our case we measure a transverse sensitivity between $20 \%$ and $30 \%$. This is in agreement with the output signal of double-sided sensor design being about $170 \%$ higher than for the one-sided sensor design. According to [16] almost every aspect of grid design and gauge construction has an effect on transverse sensitivity. One important influence could come from large ratios of width to thickness of the gridlines. Commercial strain gauges have a total thickness between 3-8 $\mu \mathrm{m}$, whereas our strain gauges have a total thickness of about 400 $\mathrm{nm}$ for the single sensor elements and $50 \mathrm{~nm}$ for the final sensor array. The width of the gridlines is nearly the same. In Figure 5 both, the influence of the sensor orientation $(l, d, t)$ and of the bending polarity (+/-) can clearly be seen for the double-sided sensor. As expected, we can see the highest sensor signal for the 
longitudinal sensor orientation, a lower signal for the diagonal orientation and the lowest signal in transverse orientation. Further, the one-sided sensor provides a slightly higher output in positive than in negative bending direction $(||+|>||-|$ and $|t+|>|t-|)$. To better visualize this effect the (I-) and (t-) curves were flipped. A possible explanation is given by a small initial curvature of the sensor foil which can be optically observed and is probably caused by different thermal expansion coefficients of SU-8 and polyimide. This initial curvature causes an initial mechanical stress and therefore an offset in the output signal, which again leads to different sensitivities for positive and negative bending. Another explanation could be a different behavior of the strain gauges for compression and for elongation, which should cancel out in the double-sided sensors. The latter is in line with absence of this effect in experiments with the double-sided sensor.

Results obtained from static measurements with the complete $6 \times 6$ sensor array which show characteristic patterns are displayed in Figure 6. The sensor foil array was bended over a test tube with a radius of $r=7.5 \mathrm{~mm}$ and output signals from all 36 sensor elements were recorded with an amplification of $A=100$. The sensor array was bent in three different modes as illustrated by the photographs.

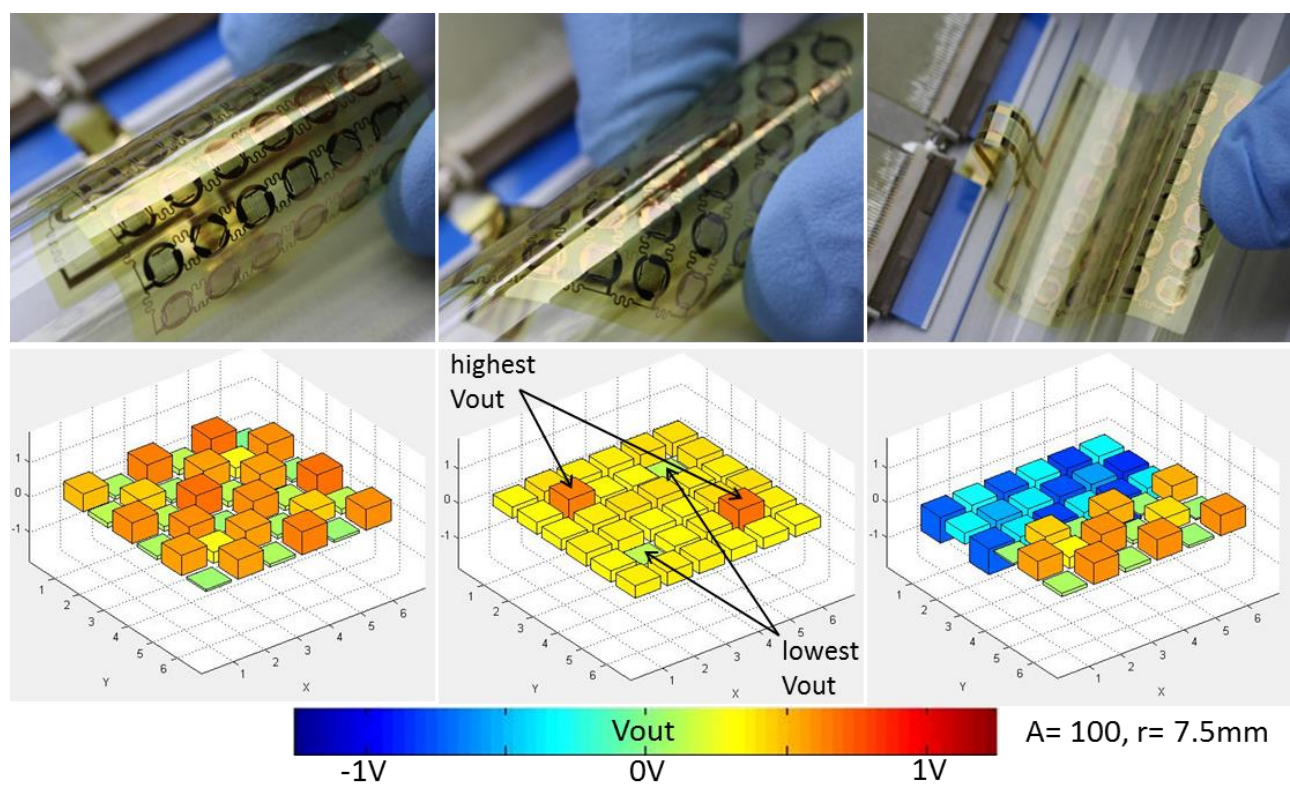

Figure 6: Bending experiments with the $6 \times 6$ sensor array (bending radius $r=7.5 \mathrm{~mm}$ ) in three different modes (bending along sensor foil edge (left), diagonal bending (middle), S-shape bending (right)) with 3D bar chart representation of the resulting 36 output signals (amplified with $A=100$ ) where the bar heights $(z)$ represent the signal strengths and the bar location $(x, y)$ the positions of the sensor elements within the array.

For the bending along the sensor foil edge alternating heights of the output signals according to the alternating sensor orientations as shown in Figure $\mathbf{3}$ can be very well recognized. For the bending in diagonal direction all longitudinally and transversally orientated sensors provide almost identical output signals because they all receive the same bending vector relative to their sensor orientation, whereas only two of the diagonally orientated sensors receive a bending in longitudinal direction and therefore provide the expected highest output signal. The two other elements receive a bending exactly in transversal direction and accordingly show the lowest output signal (sensors with lowest and highest output are marked in Figure 6). S-shaped bending signals represent very well the switch in bending polarity between the two halves of the sensor array.

In automated bending experiments the sensor array signals were analyzed in more detail as illustrated in Figure 7. This automated setup allows continuous bending of a thin sheet of metal with clamped edges by compressing the sheet with a motor-driven linear axis. The sensor array is attached by placing it between the metal sheet and a thin elastic sellotaped band (see Figure 7 upper left). The bending radius is determined optically and set to finally reach $r=20 \mathrm{~mm}$ in Figure 7 bottom left. 


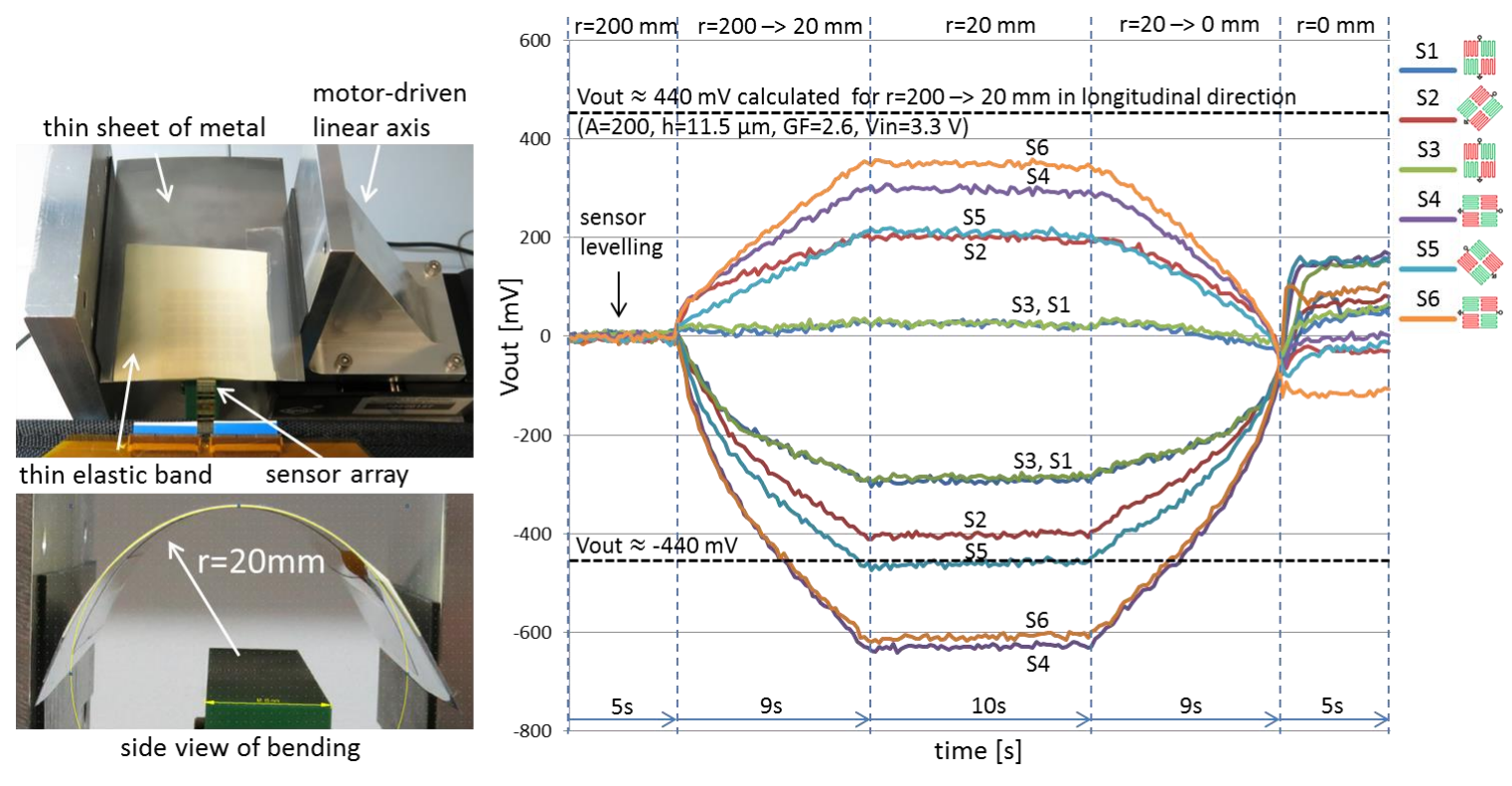

Figure 7: Automated bending experiments with the sensor array: bending/measurement setup (left), exemplary results for a measurement cycle obtained from sensors $S 1$ to $S 6$ of the $5^{\text {th }}$ column of the sensor array (right)

The measurement cycle begins with an initial bending of $r=200 \mathrm{~mm}$ and levelling of all sensors to zero. After $5 \mathrm{~s}$ at $r=200 \mathrm{~mm}$ the bending was continuously increased up to a bending radius of $r=20 \mathrm{~mm}$ remaining in this position for $10 \mathrm{~s}$ and then continuously decreased to bending radius of $r=0 \mathrm{~mm}$. The dotted lines represent calculated values of $V_{\text {out }}$ for a longitudinal bending of $r=20 \mathrm{~mm}$ (at amplification $A=200$, interlayer thickness $\mathrm{h}=11.5 \mu \mathrm{m}$, gauge factor $\mathrm{GF}=2.6$ and supply voltage of $\mathrm{V}_{\mathrm{in}}=3.3 \mathrm{~V}$ ). The gauge factor was assumed to be 2.6 for the sensor array which differs from the gauge factor of 2 assumed before for single sensor elements. The reason is that we used much thinner strain gauges for the sensor array as for the single element sensors ( $50 \mathrm{~nm}$ instead of $400 \mathrm{~nm}$ ). This difference in GF for different thicknesses of gold strain gauges is taken from $[17,18]$ and resulting calculations fit to our experimental data. The bending was applied in both polarities (+/-) and results are merged in one diagram. An immediate observation is that opposing bending polarities do not lead to symmetric values. In fact, higher amplitudes are observed in negative than in positive bending direction whereby the amplitude in negative bending exceeds the calculated value of about $-440 \mathrm{mV}$. The reason for this behavior was found out to be an initial bending in each sensor element. With an optical surface measurement out of plane deflection as shown in Figure 8 was determined and a circular fit to the line scan values revealed an initial bending radius of about $40 \mathrm{~mm}$.

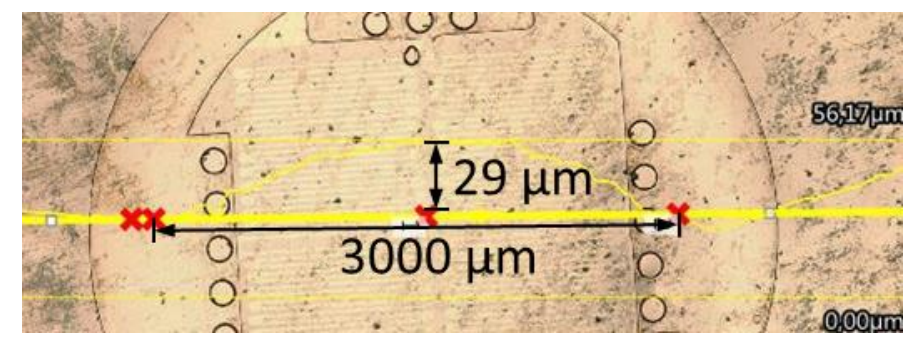

Figure 8: Optical evaluation of surface topography of a single sensor element. A line scan of $3 \mathrm{~mm}$ across a sensor element (yellow) confirms an initial out of plane deflection of $29 \mu \mathrm{m}$.

This initial bending radius of $40 \mathrm{~mm}$ was confirmed by forced flattening of the sensor array between two planar surfaces and comparing the resulting output signal with the previous relaxed state. The final state in the measurement cycle in Figure 7 shows the signals of a sensor array released to a bending radius of $r=0 \mathrm{~mm}$. With knowledge of the initial bending output signals in dependencies of sensor orientations and bending direction as displayed in Figure 7 do correlate very well with expectations. Sensor S4 and S6 are bent in longitudinal direction with the highest output signals, sensor S3 and S1 are bend in transversal direction with 
the lowest output signals and sensor S2 and S5 are bend in diagonal direction and accordingly show output signals of medium height. Some slight differences in output signal between two sensors with the same orientation can probably be also explained by the influence of initial deflections; we assume that the initial deflection slightly varies between individual sensor elements.

\section{Conclusions and outlook}

A novel flexible $6 \times 6$ sensor array on a thin foil for curvature sensing and topography reconstruction was successfully fabricated and characterized by various bending experiments. The introduced fabrication process can entirely be performed without the need to flip the substrate in between and process steps are similar to ones used in the industry already, which makes a scalable fabrication process easy to realize. A double-sided design of sensor elements improves the sensitivity by $170 \%$ and reduces signal ambiguities. Further, single sensor elements are arranged in an alternating pattern across the array allowing to fully and unambiguously determine the bending vector utilizing plausibility considerations on basis of signals from neighboring elements. Characterization results are well understood and the expectation that such sensor arrays can be used for sensor based surface reconstruction and for respiratory monitoring of premature infants seems very realistic.

In further work we will aim for decreasing the transverse sensitivity and eliminating of the initial deflection in order to provide symmetric output signals in positive and negative bending. In addition, dynamic sensor behavior, temperature influences, potentially occurring small hysteretic effects and long term mechanical and electrical stability shall be investigated to be able to utilize the sensor array as a robust and reliable device e.g. for the suggested application as respiration monitor. The sensor array shall next be turned into a stretchable system by laser cutting of the foil leaving only meandering connections between the sensor elements followed by embedding the system into PDMS or similar rubber-like material. To minimize the amount of connection lines an "active matrix" approach by integrating thin film transistors (TFTs) in the sensor design is desired. For our current design this approach would allow to reduce from 74 to 24 connection lines (12 measurement lines and 12 power supply lines) and to give the possibility to switch the power supply thereby dramatically reducing the current consumption. To optimize the read out speed of an evaluation electronic we have to consider its time consuming components.

\section{Acknowledgements}

The authors are grateful for the funding provided by the German Ministry of Economics and Technology (BMWi) under the ZIM program (Zentrales Innovationsprogramm Mittelstand) with the funding reference KF3085603TS4

\section{References}

[1] H. Schoo, System in Foil: Heterogeneous Integration, Holst Centre, available at http://www.photonics21.org/uploads/T2gZY9sg8u.pdf (accessed on April 25, 2016).

[2] J. van-den-Brand, J. de Baets, T. van Mol, A. Dietzel, Systems-in-foil-Devices, fabrication processes and reliability issues, Microelectronics Reliability (2008) 1123-1128.

[3] W.S. Wong, A. Salleo, Flexible electronics: Materials and applications, Springer, New York, London, 2008.

[4] J. van-den-Brand, M. Saalmink, M. Barink, A. Dietzel, Novel lamination and interconnection technologies demonstrated in a flexible modular optical sensor array for wound monitoring, Microelectronic Engineering (2010) 769-772.

[5] M. Schwerter, T. Beutel, M. Leester-Schädel, S. Büttgenbach, A. Dietzel, Flexible hot-film anemometer arrays on curved structures for active flow control on airplane wings, Microsystem Technologies (2014) 821-829. 
[6] G. Klink, Heterogeneous Integration on Plastic Films, Frauenhofer EMFT, available at http://www.zve-

kurse.de/Berichte.html?file=tl_files/pdf/GFE_Forum/06\%20Heterointegration\%20Technologies \%20on\%20Plastic\%20Films.pdf (accessed on 25.04.16).

[7] K. Bock, Manufactoring Technologies for System in Foil, Frauenhofer EMFT, available at http://semieurope.omnibooksonline.com/2012/semicon_europa/Plastic\%20Electronics\%20Con ference/ISS/20_Karlheinz.Bock_EMFT\%20Fraunhofer.pdf (accessed on April 25, 2016).

[8] A. Dietzel, J. van-den-Brand, J. Vanfleteren, W. Christiaens, E. Bosman, J. de Baets, Systems-inFoil Technology: Chapter 13 in Burghartz, J. (Ed.): Ultra-thin Chip Technology and Applications, Springer New York, New York, NY, 2011.

[9] G. Arutinov, E. Smits, M. Mastrangeli, G. van Heck, J. van-den-Brand, H. Schoo, A. Dietzel, Capillary self-alignment of mesoscopic foil components for sensor-systems-in-foi, J. Micromech. Microeng. 22 (2012) 115022.

[10] R. Mandamparambil, H. Fledderus, G. van Steenberge, A. Dietzel, Patterning of flexible organic light emitting diode (FOLED) stack using an ultrafast laser, Optics express 18 (2010) 7575-7583.

[11] J. van-den-Brand, R. Kusters, M. Barink, A. Dietzel, Flexible embedded circuitry: A novel process for high density, cost effective electronics, Microelectronic Engineering (2010) 1861-1867.

[12] T. Sekitani, Y. Noguchi, K. Hata, T. Fukushima, T. Aida, T. Someya, A rubberlike stretchable active matrix using elastic conductors, Science (New York, N.Y.) 321 (2008) 1468-1472.

[13] D. Waisman, A. Landesberg, S. Kohn, A. Faingersh, I.C. Klotzman, A. Gover, I. Kessel, A. Rotschild, Chest dynamics asymmetry facilitates earlier detection of pneumothorax, J Perinatol 36 (2016) 157-159.

[14] S.M. Won, H.-S. Kim, N. Lu, D.-G. Kim, C. Del Solar, T. Duenas, A. Ameen, J.A. Rogers, Piezoresistive Strain Sensors and Multiplexed Arrays Using Assemblies of Single-Crystalline Silicon Nanoribbons on Plastic Substrates, IEEE Trans. Electron Devices 58 (2011) 4074-4078.

[15] E. Koch, F. Wilsdorf, A. Dietzel, Ultra-thin sensor array for 3D curvature sensing, in: SPIE Smart Structures and Materials + Nondestructive Evaluation and Health Monitoring, San Diego, California, United States, SPIE, 2015, p. 943602.

[16] Micro-Measurements, Errors Due to Transverse Sensitivity in Strain Gages: Tech Note TN-509, available at http://www.vishaypg.com/docs/11059/tn509tn5.pdf (accessed on April 25, 2016).

[17] C. Li, P.J. Hesketh, G.J. Maclay, Thin gold film strain gauges, Journal of Vacuum Science \& Technology A 12 (1994) 813.

[18] Patent EP1749185A2 - Metallic thin film piezoresistive transduction in micromechanical and nanomechanical devices and its application in self-sensing spm probes, available at http://www.google.com/patents/EP1749185A2?cl=en\&hl=de (accessed on April 25, 2016). 